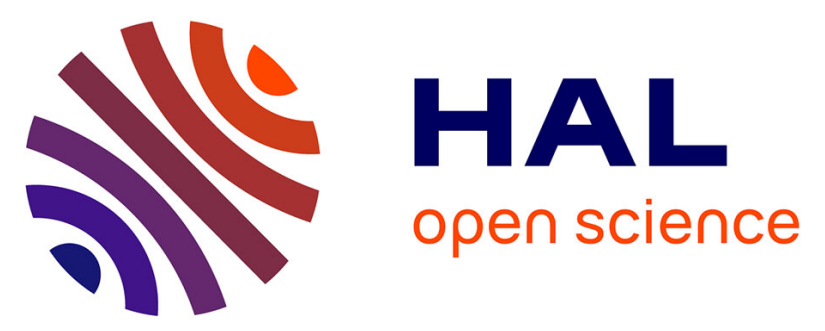

\title{
Computer-Aided Detection for Prostate Cancer Detection based on Multi-Parametric Magnetic Resonance Imaging
}

Guillaume Lemaitre, Robert Martí, Mojdeh Rastgoo, Fabrice Mériaudeau

\section{- To cite this version:}

Guillaume Lemaitre, Robert Martí, Mojdeh Rastgoo, Fabrice Mériaudeau. Computer-Aided Detection for Prostate Cancer Detection based on Multi-Parametric Magnetic Resonance Imaging. EMBC 2017 : 39th Annual International Conference of the IEEE Engineering in Medicine and Biology Society, Jul 2017, Jeju Island, South Korea. hal-01516245

\section{HAL Id: hal-01516245 \\ https://inria.hal.science/hal-01516245}

Submitted on 29 Apr 2017

HAL is a multi-disciplinary open access archive for the deposit and dissemination of scientific research documents, whether they are published or not. The documents may come from teaching and research institutions in France or abroad, or from public or private research centers.
L'archive ouverte pluridisciplinaire HAL, est destinée au dépôt et à la diffusion de documents scientifiques de niveau recherche, publiés ou non, émanant des établissements d'enseignement et de recherche français ou étrangers, des laboratoires publics ou privés. 


\title{
Computer-Aided Detection for Prostate Cancer Detection based on Multi-Parametric Magnetic Resonance Imaging
}

\author{
Guillaume Lemaître*, Robert Martí ${ }^{\dagger}$, Mojdeh Rastgoo ${ }^{\ddagger}$ and Fabrice Mériaudeau ${ }^{\S}$ \\ * Parietal team, Inria, CEA, Université Paris-Saclay, 1 Rue Honoré d'Estienne d'Orves, 91120 Palaiseau \\ $\dagger$ ViCOROB, Universitat de Girona, Campus Montilivi, Edifici P4, 17071 Girona \\ $\ddagger$ LE2I UMR6306, CNRS, Arts et Métiers, Univ. Bourgogne Franche-Comté, 12 rue de la Fonderie, 71200 Le Creusot \\ $\S$ CISIR, Electrical \& Electronic Engineering Department,Universiti Teknologi PETRONAS, 32610 Seri Iskandar, Perak \\ I Corresponding author: fabrice.meriaudeau@utp.edu.my
}

\begin{abstract}
Prostate cancer $(\mathrm{CaP})$ is the second most diagnosed cancer in men all over the world. In the last decades, new imaging techniques based on magnetic resonance imaging (MRI) have been developed improving diagnosis. In practice, diagnosis is affected by multiple factors such as observer variability and visibility and complexity of the lesions. In this regard, computer-aided detection and diagnosis (CAD) systems are being designed to help radiologists in their clinical practice. We propose a CAD system taking advantage of all MRI modalities (i.e., $\mathbf{T}_{2}$-W-MRI, DCE-MRI, diffusion weighted (DW)-MRI, MRSI). The aim of this CAD system was to provide a probabilistic map of cancer location in the prostate. We extensively tested our proposed CAD using different fusion approaches to combine the features provided by each modality. The source code and the dataset have been released.
\end{abstract}

\section{INTRODUCTION}

Current prostate cancer $(\mathrm{CaP})$ screening consists of 3 different stages. First, prostate-specific antigen (PSA) control is performed to distinguish between low- and high-risk $\mathrm{CaP}$. To assert such diagnosis, samples are taken during prostate biopsy and analyzed to make an accurate prognosis of the $\mathrm{CaP}$.

Although PSA screening has been shown to improve early detection of $\mathrm{CaP}$ [1], its lack of reliability motivates further investigations using magnetic resonance imaging (MRI)-based computer-aided detection and diagnosis (CAD). Consequently, current research is focused on identifying new biological markers to replace PSA-based screening [2]. Until such research comes to fruition, these needs can be met through active-surveillance strategy using multiparametric MRI (mp-MRI) techniques [3].

Lemaitre $e t$ al. recently reviewed more than 50 research works that focused on CAD system for CaP [4]. These studies are based on CAD systems that consist of the following steps: (i) pre-processing, (ii) segmentation, (iii) registration, (iv) feature detection, (v) feature selection-extraction, and (vi) finally classification.

The reviewed mp-MRI-based CAD used 2 to 3 MRI modalities among $\mathrm{T}_{2}$ Weighted $\left(\mathrm{T}_{2}\right.$-W)-MRI, dynamic contrast-enhanced (DCE)-MRI, and diffusion weighted (DW)-MRI, discarding the potential discriminative power of magnetic resonance spectroscopy imaging (MRSI). Furthermore, only half of these studies tackled the challenging detection of $\mathrm{CaP}$ in the central gland (CG). Additionally, none of the works investigated the issue related to feature balancing when developing their CAD systems. Finally, none of the datasets nor source codes used have been released, making impossible the possibilities to compare the methods.

In this work, we propose a CAD system to detect $\mathrm{CaP}$ in peripheral zone (PZ) and CG, using the 4 aforementioned MRI modalities. The ultimate goal of the current CAD system is to provide a probabilistic map of the cancer within the prostate. Therefore, each voxel in the prostate will be classified as healthy or cancerous. The dataset used and the source code developed are released for future comparisons and reproducibility.

\section{Methodology}

\section{A. Materials}

The mp-MRI data are acquired from a cohort of patients with higher-than-normal level of PSA. Acquisition is achieved with a $3 \mathrm{~T}$ whole body MRI scanner (Siemens Magnetom Trio TIM, Erlangen, Germany) using sequences to obtain $\mathrm{T}_{2}$-W-MRI, DCE-MRI, DW-MRI, and MRSI. In addition of the MRI examination, these patients also have undergone a transrectal ultrasound (TRUS) guided-biopsy. The dataset is composed of 17 which all have biopsies that were positive for $\mathrm{CaP}$. From those 12 patients have a $\mathrm{CaP}$ in the $\mathrm{PZ}, 3$ patients have $\mathrm{CaP}$ in the $\mathrm{CG}, 2$ patients have invasive $\mathrm{CaP}$ in both the $\mathrm{PZ}$ and the $\mathrm{CG}$. An experienced radiologist segmented the prostate organ - on $\mathrm{T}_{2}$-W-MRI, DCE-MRI, and apparent diffusion coefficient (ADC) - as well as the prostate zones - i.e., $\mathrm{PZ}$ and $\mathrm{CG}-$, and $\mathrm{CaP}$ on the $\mathrm{T}_{2}$-W-MRI. The full description and the data set are available at $\mathrm{I} 2 \mathrm{Cvb}$ website ${ }^{1}[5]$.

\section{B. CAD pipeline for $\mathrm{CaP}$}

Our mp-MRI CAD system consists of 7 different steps: pre-processing, segmentation, registration, feature detection, feature balancing, feature selection/extraction, and finally classification. The different source codes are publicly available ${ }^{2}$.

\footnotetext{
${ }^{1}$ http://i2cvb.github.io/

${ }^{2}$ https://github.com/I2Cvb/mp-mri-prostate
} 
1) Pre-processing: Normalization is, a crucial step to reduce the inter-patient variations which allows to improve the learning during the classification stage. However, the MRI modalities provide specific type of data - static vs. dynamic information, images $v s$. signals - that required a dedicated pre-processing. Therefore, we pre-process differently the data: $\mathrm{T}_{2}-\mathrm{W}$-MRI is normalized using a Rician apriori that has been shown to be better than the traditional $z$-score [6]. In contrast to $\mathrm{T}_{2}-\mathrm{W}$-MRI, in ADC map the probability density function (PDF) within the prostate does not follow a known distribution and thus one cannot use a parametric model to normalize these images and a nonparametric piecewise-linear normalization [7] is the best option for this case. DCE-MRI is a dynamic sequence and the data are normalized based on a mean kinetic expression registration as proposed in [5]. Finally, the MRSI modality has been pre-processed to correct the phase, suppress the baseline, and align the frequencies [8].

2) Segmentation and registration: For this work, our radiologist has manually segmented the prostate organs on the different modalities. However, the segmented prostate needs to be registered before to extract features. Therefore, the patients motion during the DCE-MRI is corrected using a rigid registration with an mean squared error (MSE) similarity metric and a gradient descent optimizer. Subsequently, the $\mathrm{T}_{2}-\mathrm{W}-\mathrm{MRI}$ and DCE-MRI are co-registered using a rigid transformation and the delineation of the prostate gland, using the same metric and optimizer previously mentioned. ADC maps and $\mathrm{T}_{2}-\mathrm{W}$-MRI are also co-registered with the same strategy. Additionally, volumes from all modalities have been interpolated to the resolution of $\mathrm{T}_{2}-\mathrm{W}$-MRI.

3) Feature detection: Similarly to the pre-processing, specific features are extracted depending of the specificity of each MRI modality.

$\mathbf{T}_{2}$-W-MRI and ADC map features Additionally to the normalized intensity, edge- and texture-based features are commonly extracted from $\mathrm{T}_{2}-\mathrm{W}-\mathrm{MRI}$ and $\mathrm{ADC}$ map. The following set of filters characterizing edges have been used: (i) Kirsch, (ii) Laplacian, (iii) Prewitt, (iv) Scharr, (v) Sobel, and (vi) Gabor. Except for the Kirsch filter, the other filters are applied in 3D, taking advantage of the volume information instead of slice information, as it is usually done. Additionally, features based on phase congruency are computed [9]. To characterize the local texture, both second-order graylevel co-occurence matrix (GLCM)-based features [10] and rotation invariant and uniform local binary pattern (LBP) [11] are extracted. To encode 3D information, the 13 first Haralick features are computed for the 13 possible directions. For the same reason, the LBP codes are computed for the three-orthogonal-planes of each MRI volume. All these features are extracted at each voxel of the volume.

DCE-MRI features In brief, the entire enhanced signal, semi-quantitative [12], and quantitative-based models [13], [14], [15], [16] are computed.

MRSI features Three different techniques are used to ex- tract discriminative features: (i) relative quantification based on metabolite quantification, (ii) relative quantification based on bounds integration, and (iii) spectra extraction from $2 \mathrm{ppm}$ to $4 \mathrm{ppm} \mathrm{[5].}$

Anatomical features Four different metrics are computed based on the relative distance to the prostate boundary as well as the prostate center, and the relative position in the Euclidean and cylindrical coordinate systems [17], [18].

4) Feature balancing: Imbalanced dataset is a common problem in medical imaging. The number of cancerous voxels is much lower than the number of "healthy" voxels for a patient. This problem compromises the learning process. Solving the problem of imbalanced is equivalent to under- or over-sampling part of the dataset to obtain equal number of samples in both classes. In this regard, the imbalanced dataset was under-sampled using the different variant of nearmiss (NM) [19] and the instance-hardnessthreshold (IHT) [20] algorithm. In addition, the dataset was also balanced using over-sampling methods, namely different variant of synthetic minority over-sampling techniques (SMOTE) [21], [22]. Those algorithms were developed and made publicly available in the scikit-learn-contrib imbalanced-learn ${ }^{3}$ python package [23].

5) Feature selection and extraction: Feature selection and extraction are used in our experiment. MRSI and DCEMRI are decomposed using three feature extraction methods: principal components analysis (PCA), sparse-PCA, and independent components analysis (ICA). Additionally to feature extraction, two methods of feature selection are used: (i) the one-way analysis of variance (ANOVA) and (ii) the Gini importance obtained while learning the random forest (RF) classifiers. The scikit-learn ${ }^{4}$ python package provides all those methods and was used in our experiment [24].

6) Classification: RF has been chosen as our base classifier to perform classification of individual modality as well as the combination of modalities. RF and decision trees do not require to scale features and provide feature selection by analyzing the feature importance, derived from the impurity improvement successive splits. Additionally, we use stacking to create ensemble of base learners using a meta-classifier [25], namely AdaBoost (AdB) and Gradient Boosting (GB).

\section{RESULTS AND EVALUATION}

Various experiments were run in order to optimize the balancing and the feature selection strategies [5]. We found that once all features are concatenated together, nearmiss-3 (NM-3) [19] is the method providing the best enhancement of the classification performance with an area under the curve (AUC) of $0.824 \pm 0.076$. Therefore, with this optimal balancing, we here report the final step consisting of three strategies: (i) the selected features from each modality (i.e.,

\footnotetext{
${ }^{3}$ http: //contrib.scikit-learn.org/ imbalanced-learn/

${ }^{4}$ http://scikit-learn.org/stable/
} 
TABLE I

SELECTED FEATURE AND NUMBER OF OCCURRENCE FOR $\mathrm{T}_{2}$-W-MRI, ADC MAP, AND ONE ALL THE FEATURES ARE CONCATENATED.

\begin{tabular}{|c|c|c|c|c|c|}
\hline $\mathbf{T}_{2}$-W-MRI & ADC & $\mathbf{T}_{2}$-W-MRI & ADC & DCE-MRI & MRSI \\
\hline $\begin{array}{l}8 \text { edges } \\
155 \text { Gabor filters } \\
2 \text { Haralick features } \\
1 \text { intensity } \\
4 \text { LBP } \\
2 \text { phase congruency }\end{array}$ & $\begin{array}{l}1 \text { DCT } \\
32 \text { Gabor filters } \\
1 \text { phase congruency }\end{array}$ & $\begin{array}{l}113 \text { Gabor filters } \\
1 \text { phase congruency } \\
4 \text { edges } \\
1 \text { intensity }\end{array}$ & $\begin{array}{l}53 \text { Gabor filters } \\
2 \text { phase congruency }\end{array}$ & 14 samples & 78 samples \\
\hline 172 features & 34 features & & 267 features & & \\
\hline
\end{tabular}

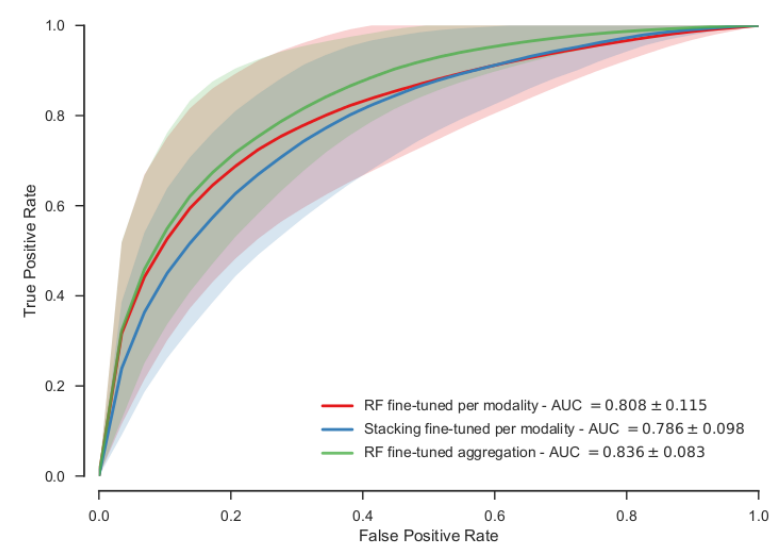

Fig. 1. Analysis of feature combination approaches after fine tuning through balancing and feature selection/extraction.

331 features) are concatenated together and used in a RF classifier, (ii) the selected features from each modality (i.e., 331 features) are used to train a stacking classifier with a $\mathrm{GB}$ as meta-classifier, and (iii) the selected features from the concatenated set of features (i.e., 267 features) are used to train a single RF classifier.

The selected features are presented in Table I which highlights some interesting facts regarding the most efficient features. On the one hand, the Gabor filters and the phase congruency are always selected, independently of the strategy and modality during the feature selection process. Additionally, edge filters - i.e., Kirsch, Prewitt, Scharr, and Sobel — have been only selected for the $\mathrm{T}_{2}$-W-MRI. A possible explanation might be due to the fact that $\mathrm{T}_{2}-\mathrm{W}$ MRI is the modality with the highest spatial resolution and in which the level of details is the most important. Subsequently, the intensity feature of the $\mathrm{T}_{2}$-W-MRI modality is always selected, implying that our normalization method proposed in [6] is efficient.

The experiments were performed in a leave-one-patientout cross-validation (LOPO CV) fashion and a receiver operating characteristic (ROC) analysis is carried out. The comparative results are shown in Fig. 1. In overall, classification using the fine-tuned features improve the classification performance. The third classification configuration is, however, the one which outperforms others with an AUC of $0.836 \pm 0.083$. The improvement in terms of AUC is of 0.028 and 0.050 compared with the $1^{\text {st }}$ and $2^{\text {nd }}$ configurations,

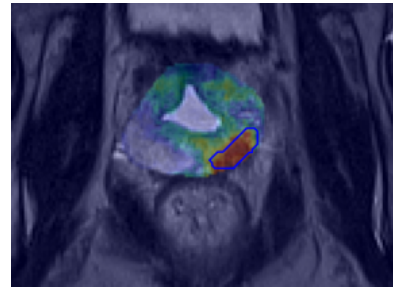

(a) $\mathrm{AUC}=0.922$

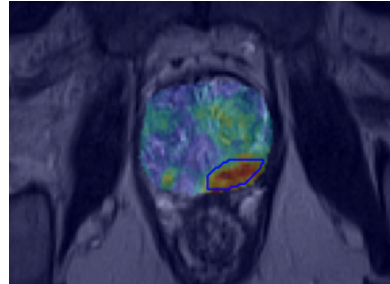

(b) $\mathrm{AUC}=0.914$
Fig. 2. Illustration the resulting detection of our mp-MRI CAD for $\mathrm{CaP}$ detection. The blue contours corresponds to the $\mathrm{CaP}$ while the jet overlay represents the probability.

respectively.

In a clinical setting, the AUC score is categorized in 3 levels: (i) "acceptable" discrimination for an AUC ranging from 0.7 to 0.8 , (ii) "excellent" discrimination for an AUC ranging from 0.8 to 0.9 , and "outstanding" discrimination when the AUC is over 0.9 [26]. Therefore, the combination of all MRI modalities in conjunction with fine-tuning allow to upgrade our CAD system from an "acceptable" to an "excellent" discrimination level.

To illustrate qualitatively the results of our mp-MRI CAD system, 2 diverse examples are presented in Fig. 2 by overlapping the probability map of having a $\mathrm{CaP}$ with the original $\mathrm{T}_{2}$-W-MRI slice.

\section{CONCLUSION}

In this paper, we presented one of the the first CAD system using all the mp-MRI modalities for prostate cancer detection. Indeed, MRSI has nearly never been used together with the other modalities. With an extensive validation approach to select the best features, the best balancing strategy as well as the best classifier, we obtained results on a rather complicated dataset of 17 patients with an average AUC of $0.836 \pm 0.083$ which put our system in the state-of-the-art, even so different CADs were tested on different datasets. In addition, we released the source code and the dataset allowing for reproducibility.

As avenues for future research, one could switch from voxel-based classification to super-voxel classification such that spatial structure are classified instead of voxel. In addition, the registration relies on the segmentation of the prostate gland which was provided by our doctors. To be 
used in a clinical environment, this step need to be fully automatized.

\section{REFERENCES}

[1] R. Chou, J. M. Croswell, T. Dana, C. Bougatsos, I. Blazina, R. Fu, K. Gleitsmann, H. C. Koenig, C. Lam, A. Maltz, J. B. Rugge, and K. Lin, "Screening for prostate cancer: a review of the evidence for the U.S. Preventive Services Task Force," Ann. Intern. Med., vol. 155, no. 11, pp. 762-771, Dec 2011.

[2] J. Brenner, A. Chinnaiyan, and S. Tomlins, "ETS fusion genes in prostate cancer," in Prostate Cancer, ser. Protein Reviews, D. J. Tindall, Ed. Springer New York, 2013, vol. 16, pp. 139-183.

[3] C. M. Moore, A. Ridout, and M. Emberton, "The role of MRI in active surveillance of prostate cancer," Curr Opin Urol, vol. 23, no. 3, pp. 261-267, May 2013.

[4] G. Lemaitre, R. Marti, J. Freixenet, J. C. Vilanova, P. M. Walker, and F. Meriaudeau, "Computer-aided detection and diagnosis for prostate cancer based on mono and multi-parametric mri: A review," Computers in Biology and Medicine, vol. 60, pp. 8-31, 2015.

[5] G. Lemaitre, "Computer-Aided Diagnosis for Prostate Cancer using Multi-Parametric Magnetic Resonance Imaging," Ph.D. dissertation, Universitat de Girona and Université de Bourgogne, 2016.

[6] G. Lemaitre, M. R. Dastjerdi, J. Massich, J. C. Vilanova, P. M. Walker, J. Freixenet, A. Meyer-Baese, F. Mériaudeau, and R. Marti, "Normalization of $\mathrm{t} 2 \mathrm{w}$-mri prostate images using rician a priori," in SPIE Medical Imaging. International Society for Optics and Photonics, 2016, pp. 978 529-978 529.

[7] L. G. Nyul, J. K. Udupa, and X. Zhang, "New variants of a method of MRI scale standardization," IEEE Trans Med Imaging, vol. 19, no. 2, pp. 143-150, Feb 2000.

[8] S. Parfait, P. Walker, G. Crhange, X. Tizon, and J. Mitran, "Classification of prostate magnetic resonance spectra using Support Vector Machine ," Biomedical Signal Processing and Control, vol. 7, no. 5, pp. $499-508,2012$.

[9] P. Kovesi, "Image features from phase congruency," Videre: Journal of computer vision research, vol. 1, no. 3, pp. 1-26, 1999.

[10] R. Haralick, K. Shanmugam, and I. Dinstein, "Textural features for image classification," Systems, Man and Cybernetics, IEEE Transactions on, vol. SMC-3, no. 6, pp. 610-621, 1973.

[11] T. Ojala, M. Pietikainen, and T. Maenpaa, "Multiresolution gray-scale and rotation invariant texture classification with local binary patterns," IEEE Transactions on pattern analysis and machine intelligence, vol. 24, no. 7, pp. 971-987, 2002.

[12] H. J. Huisman, M. R. Engelbrecht, and J. O. Barentsz, "Accurate estimation of pharmacokinetic contrast-enhanced dynamic MRI parameters of the prostate," J Magn Reson Imaging, vol. 13, no. 4, pp. 607-614, Apr 2001
[13] G. Brix, W. Semmler, R. Port, L. R. Schad, G. Layer, and W. J. Lorenz, "Pharmacokinetic parameters in cns gd-dtpa enhanced mr imaging." Journal of computer assisted tomography, vol. 15, no. 4, pp. 621-628, 1991.

[14] U. Hoffmann, G. Brix, M. V. Knopp, T. He $\beta$, and W. J. Lorenz, "Pharmacokinetic mapping of the breast: a new method for dynamic mr mammography," Magnetic resonance in medicine, vol. 33, no. 4 , pp. 506-514, 1995

[15] P. S. Tofts, B. Berkowitz, and M. D. Schnall, "Quantitative analysis of dynamic gd-dtpa enhancement in breast tumors using a permeability model," Magnetic Resonance in Medicine, vol. 33, no. 4, pp. 564-568, 1995.

[16] V. Giannini, S. Mazzetti, A. Vignati, F. Russo, E. Bollito, F. Porpiglia, M. Stasi, and D. Regge, "A fully automatic computer aided diagnosis system for peripheral zone prostate cancer detection using multi-parametric magnetic resonance imaging," Computerized Medical Imaging and Graphics, vol. 46, pp. 219-226, 2015.

[17] L. Chen, Z. Weng, L. Goh, and M. Garland, "An efficient algorithm for automatic phase correction of $\{\mathrm{NMR}\}$ spectra based on entropy minimization ," Journal of Magnetic Resonance, vol. 158, no. 12, pp. $164-168,2002$.

[18] G. Litjens, O. Debats, J. Barentsz, N. Karssemeijer, and H. Huisman, "Computer-iided detection of prostate cancer in MRI," Medical Imaging, IEEE Transactions on, vol. 33, no. 5, pp. 1083-1092, May 2014.

[19] I. Mani and I. Zhang, "knn approach to unbalanced data distributions: a case study involving information extraction," in Proceedings of Workshop on Learning from Imbalanced Datasets, 2003.

[20] M. R. Smith, T. Martinez, and C. Giraud-Carrier, "An instance level analysis of data complexity," Machine learning, vol. 95, no. 2, pp. 225-256, 2014.

[21] N. V. Chawla, K. W. Bowyer, L. O. Hall, and W. P. Kegelmeyer, "Smote: synthetic minority over-sampling technique," Journal of artificial intelligence research, pp. 321-357, 2002.

[22] H. Han, W. Y. Wang, and B. H. Mao, "Borderline-smote: a new oversampling method in imbalanced data sets learning," in International Conference on Intelligent Computing. Springer, 2005, pp. 878-887.

[23] G. Lemaître, F. Nogueira, and C. K. Aridas, "Imbalanced-learn: A python toolbox to tackle the curse of imbalanced datasets in machine learning," Journal of Machine Learning Research, vol. 18, no. 17, pp. 1-5, 2017. [Online]. Available: http://jmlr.org/papers/v18/16-365.html

[24] F. Pedregosa, G. Varoquaux, A. Gramfort, V. Michel, B. Thirion, O. Grisel, M. Blondel, P. Prettenhofer, R. Weiss, V. Dubourg et al., "Scikit-learn: Machine learning in python," Journal of Machine Learning Research, vol. 12, no. Oct, pp. 2825-2830, 2011.

[25] D. H. Wolpert, "Stacked generalization," Neural networks, vol. 5 , no. 2, pp. 241-259, 1992.

[26] D. W. Hosmer Jr and S. Lemeshow, Applied logistic regression. John Wiley \& Sons, 2004. 